\title{
Neuropsychological Model of the Realisation of Information Need
}

\author{
Yashar Moshfeghi \\ University of Strathclyde \\ Frank E. Pollick \\ University of Glasgow
}

\begin{abstract}
The main goal of Information Retrieval (IR) is to satisfy information need (IN). IN refers to a complex concept: at the very initial state of the phenomenon (i.e. at a visceral level), even the searcher may not be aware of its existence. Thus despite advances in the past few decades in both the IR and relevant scientific communities, we do not fully understand how an IN emerges and how it is physically manifested. In this paper, we aim to inform a holistic view of the realisation of IN using functional Magnetic Resonance Imaging (fMRI).

We collected new data of brain activity of twenty-four participants while they formulated and stated a realisation of IN in a Question Answering task, focusing on a distributed set of brain regions associated with activities related to information need, found in our previous study. Results of a functional connectivity analysis led us to propose a neuropsychological model of the realisation of IN. Our model consists of three components: (i) a successful memory retrieval component, (ii) an information flow regulation component, and (iii) a high-level perception component. We believe this study constitutes an important step in unravelling the nature of IN and how to better satisfy IN.
\end{abstract}

Keywords: Information Need, Information Retrieval, fMRI Study, User Study

Information Need (IN) plays a fundamental role in an information seeking and retrieval process, yet, due to the complexity associated with it (Cole, 2011), it is one of the most difficult concepts in Information Retrieval (IR) to understand, capture, and in turn, satisfy. Over the last several decades much research has been dedicated to shedding light on this concept in both IR (Belkin, Oddy, \& Brooks, 1982a; Ingwersen, 1996; Wilson, 1981) and other relevant scientific communities such as Information Science (Cole, 2011). This research has led to the publication of seminal theories, models, and findings that have shaped the foundations of current IR systems. Examples of such influential works are Wilson's Information Seeking Behaviour model (Wilson, 1981), Kuhlthau's Information Seeking 
Process (ISP) model (Kuhlthau, 1993), Ingwersen's Cognitive IR Theory (Ingwersen, 1996), and Belkin's Anomalous States of Knowledge (ASK) model (Belkin et al., 1982a). These works are based on the behavioural study of searchers while they engage in an Information Retrieval and Seeking process, and have mainly involved questionnaires/interviews (Kuhlthau, 1993), or by observing and studying searchers interaction with IR systems via their submitted queries and their reformulation (Kelly \& Fu, 2007), or via their interaction with retrieved results (White, 2004). Despite the many invaluable contributions, they have all investigated the phenomenon of IN indirectly, via some mediator. In this research, we use brain imaging to obtain a new perspective on understanding the mechanisms of information need.

\section{IN Complexity and the IN-Query Gap}

Information need is an essential concept that is at the core of the IR processes. When searchers realise an IN, they experience an anomaly in their current state of knowledge (ASK) (Belkin et al., 1982a). As a result, search processes are initiated: searchers transform their IN into a query and submit it to an IR system. In turn, the IR system retrieves potentially relevant documents, aiming to satisfy the IN. Subsequently, searchers evaluate retrieved documents, accumulating relevant information which leads them to satisfy their IN. Often, however, searchers are not satisfied with the results obtained in response to their initial query formulation (van Rijsbergen, 1986), and thus must engage in further interaction with the system to resolve their need. Therein lies the complexity associated with the concept of IN.

The complexity of IN rests in its paradoxical nature (Cole, 2011): Unlike other primary human needs (e.g., physiological ones), it is often unknown to the individual beforehand what information is required to satisfy their IN. Research indicates that this is because an IN is "intangible and visceral" and therefore "unknowable and non-specifiable" - thus, how can one express an IN using a query to an IR system? (Belkin et al., 1982a; Cole, 2011) Hence, the very nature of IN inherently makes it nearly impossible for searchers to correctly map their IN to an IR query (Belkin et al., 1982a, 1982b; Cole, 2011; Hjørland, 2010; Taylor, 1968). This produces a gap between how an IN is represented (i.e. the formulated query) and the actual IN because the formulated query is not guaranteed to provide an exact description required to retrieve the relevant documents (White, Jose, \& Ruthven, 2003). In other words, expressing an IN using a set of query keywords is considered to be uncertain and noisy (Ingwersen \& Järvelin, 2006), as it can only vaguely approximate the actual IN (Taylor, 1968). The problem becomes even more pronounced when an IN is "illdefined": i.e., when the searcher only knows "fringes of a gap in [his/her] knowledge" (Cole, 2011) making it extremely difficult for the searcher to identify and describe the IN (Bates, 1998; Belkin et al., 1982a; Borgman, 2000). Therefore, it is possible that a given query may not sufficiently define the characteristics of relevant documents, or even any relevant information since a searcher cannot form an appropriate initial state from which to form a query (Cole, Julien, \& Leide, 2010).

Taylor (1968)'s classic four-level theory of IN theorised the complex nature of IN as follows: (i) a search begins with a process based on an area of doubt or a gap in understanding, which is a compromised expression of the need (Taylor calls this the Q4 level). The searcher, as a result of searching, can subsequently frame an IN, borrowing generic 
knowledge frames from adjacent areas in memory; at a certain point, a transformational event of information use leads to the IN being actualised, resulting in the information search becoming focused. This transformation in the use of information causes the searcher's Q4 compromised level of need to access deeper levels of the need, i.e. the formal expression of the need (Taylor calls this the Q3 level), the conscious "within brain" description of the need (Taylor calls this the Q2 level) and finally the deepest level of the need is the "visceral" (i.e., instinctual) level (Taylor calls the Q1 level) (Cole, 2011; Taylor, 1968). What is of particular interest to the current paper are the lower levels in Taylor's conceptualisation of IN, namely the unconscious, visceral information need (which the user cannot know and therefore cannot specify to the IR system) leading to the "within brain" description of the IN.

Ingwersen (1996) in his Cognitive theory of IR explains that, based on the perspective of cognitive science, theories of information and empirical evidence, an appropriate approach to understanding IR is first to examine the mental formation of the information need and use this as the starting point for IR interaction. This formulation is a central issue in IR. This paper takes substantial motivation from this vein of research, arguing that IR systems that can detect and understand INs, starting from their Q1 levels, can better satisfy them.

\section{Neuroscience and IR}

Research into the neural bases of IR is becoming established as an important perspective with which to understand IR (Gwizdka \& Mostafa, 2016) and this is reflected in the growing body of literature that employs a variety of brain imaging techniques. Much of this work has been directed towards understanding relevance. For example, M. J. Eugster et al. (2014) used EEG to show that the frequency content of the EEG signal as well as Event-Related Potentials (ERPs) can be used to define a set of features that enable decoding whether a text is relevant. This work was later extended to demonstrate how the relevance of a written word could be identified from the EEG activity appearing 200-950 ms following it being read (M. J. A. Eugster et al., 2016). Consistent with these works, Allegretti et al. (2015) used EEG to show that within 500 ms EEG signals begin to appear that differentiate between viewing a relevant and a non-relevant image. Similarly, Kauppi et al. (2015) used magnetoencephalography (MEG) to show that the frequency content of the MEG signal, along with eye movement data can be used to decode whether viewed images were relevant. The combination of eye movement data and EEG signal was further explored in the domain of successfully identifying the relevance of reading text (Gwizdka, Hosseini, Cole, \& Wang, 2017). While techniques such as EEG and MEG have a higher temporal resolution, fMRI provides higher spatial resolution that enables localising where in the brain activity is occurring. Along these lines, a study by Moshfeghi, Pinto, Pollick, and Jose (2013) used fMRI to identify brain regions activated by the process of judging the relevance of an image. These brain regions included the inferior parietal lobe, inferior temporal gyrus and superior frontal gyrus and the increased activation revealed for viewing relevant items were related to visuospatial working memory.

Information need and the ability to resolve the need appears to be a complex, high level mental phenomenon that is built upon elemental psychological systems such as memory retrieval, attention, decision making and executive function. Thus, we can expect studies of information need to have common ground with these more elemental psychological processes. 
In addition, one high level psychological process that appears similar to information need is known as metacognition (Flavell, 1979). Metacognition generally refers to higher order thinking that involves active control over, and assessment of the cognitive processes involved in learning and storing information . A guiding framework of metacognition was provided by Nelson and Narens (1990) that divided metacognition into a combination of monitoring and control processes. One such monitoring process, known as "feeling of knowing" (FOK), resonates with the problem of information need as it involves an individual indicating that they cannot currently answer a question but can provide a judgment (e.g. confidence) about whether they could potentially recall the answer in the future.

There has been considerable interest in cognitive neuroscience to understand the neural basis of metamemory and metacognitive abilities in general (Fleming \& Dolan, 2012). A review of metamemory research (Chua, Pergolizzi, \& Weintraub, 2014) indicated for FOK the involvement of various regions in the prefrontal cortex. For example, Kikyo, Ohki, and Miyashita (2002) found activations in left dorsolateral, left anterior, bilateral inferior, and medial prefrontal cortices significantly increased with increasing reports of FOK. Moreover, they demonstrated that regions associated with FOK in the right inferior frontal gyrus and a subset of the FOK region in the left inferior frontal gyrus were not recruited for successful recall processes, suggesting their particular role in metamemory processing. In addition to the involvement of frontal cortex in FOK there is also evidence for activity in the posterior parietal cortex being modulated by the degree of FOK (Maril, Simons, Mitchell, Schwartz, \& Schacter, 2003).

In the current research, we examine neural correlates of information need and extend a study by Moshfeghi, Triantafillou, and Pollick (2016) that examined two scenarios where brain activity was contrasted between when searchers had an information need and when they did not. In one scenario when searchers had an information need they simply indicated the need to search without engaging in a search process (Question-Response (QR) task Scenario 1) and in the other scenario when searchers realised an information need they later engaged in a search process (Question-Response-Search (QRS) task- Scenario 2). For both the QR and QRS task brain activity was contrasted between when there was information need versus when there wasn't. For the QR task, results showed higher activity in the left inferior frontal gyrus and several subcortical regions when there was no information need and higher activity in ventral posterior cingulate when there was an information need. Results for the QRS task showed higher activity with information need in the fusiform, cuneus and the dorsal posterior cingulate. The change between ventral and dorsal posterior cingulate activity was interpreted in the context of the Attention, Breadth and Balance (ABBA) model (Leech \& Sharp, 2014) as being consistent with a switch from a narrow internal focus to a broad external focus of attention. In the current study, we revisit the results of Moshfeghi et al. (2016) to examine the interaction between these previously reported regions, extending this analysis to include the full search and document evaluation times that followed the QRS task.

In order to assess the interaction between brain regions revealed from the results of Moshfeghi et al. (2016) we measure functional connectivity. Functional connectivity measures the correlation in brain activity between different brain regions (Friston, 2011). Although measures of functional connectivity among a set of brain areas are limited by not specifying a direction of influence between the brain regions and being blind to brain regions 
outside the set of considered regions that might be a common cause of the correlation among the considered set of areas, it does provide evidence of whether the set of brain regions form a network. The results of Moshfeghi et al. (2016) provide us with brain regions that appear sensitive to IN, and we are interested in the general interaction structure revealed by functional connectivity of these regions. In particular, one set of brain regions was found to be more active when there was an IN realisation, and a different set of brain regions was found to be more active when there was not an IN realisation. One question we address is whether these two sets of brain regions appear independent, or whether there is an interplay between these IN and No-IN regions. Another question we address is whether there is a difference between the functional connectivity obtained in the two scenarios studied by Moshfeghi et al. (2016). In one of the scenarios individuals engaged in a search after stating an information need (Don't know - Do Search Scenario) in the other scenario individuals simply stated the need to search and then advanced to the next trial (Don't know - Don't search Scenario). Thus, our research addresses the following research questions:

- RQ1: "In each scenario in isolation, is there a functional connectivity between any of the regions reported by Moshfeghi et al. (2016)?"

- RQ2: "Is there a functional connectivity between regions reported for IN and regions reported for No-IN, in each scenario in isolation, by Moshfeghi et al. (2016)?"

- RQ3: "How do the functional connectivities obtained for the regions reported for Scenario 1 and for Scenario 2 by Moshfeghi et al. (2016), differ?"

An answer to these questions will improve our understanding of the IN concept and can lead us to develop more holistic and robust models of IN from a neuropsychology point of view. Such a model, fundamentally, will play a key role in opening new doors to the design and implementation of novel IR techniques that will enable better and even possibly proactive means to satisfy a searchers' need. The research described in this paper represents our efforts in this direction.

The remainder of the paper is organised as follows: Related Work Section presents related work and the background. Experimental Methodology Section discusses at length the experimental methodology. Results Section presents and analyses our results. Information Need Realisation Model Section presents our neuropsychology- inspired IN realisation model. Finally, Discussion and Conclusion Section presents our key conclusions.

\section{Experimental Methodology}

\section{Participants}

We have recruited twenty-four healthy participants for this study. All participants were under the age of 44, with the largest group between the ages of 18-23 (54.1\%) followed by a group between the ages of 30-35 (20.8\%). The Edinburgh handedness questionnaire (Oldfield, 1971) indicated that $79.1 \%$ were right-handed, $12.5 \%$ were left-handed, and $8.33 \%$ were mixed-handed. Participants tended to have a postgraduate degree $(20.8 \%)$, bachelors $(33.33 \%)$ or other qualifications $(45.8 \%)$. They were primarily students $(54.1 \%)$, though there were a number of individuals who were self-employed $(20.8 \%)$, not employed $(4.16 \%)$ or employed by a company or organisation (20.8\%). Participants were primarily native speakers $(79.1 \%)$ or had an advanced level of English (20.8\%). They all had experience in searching, with an average of 11.66 years (SD of 3.58) experience. 


\section{Design}

A "within-subjects" design was used in this study, and the dependent variable was the calculation of functional brain connectivity from BOLD signal during two different scenarios. To address RQ1 and RQ2, we examine the structure of the networks obtained for each scenario and to address RQ3 we compare the network obtained for Scenario 1 to the network obtained for Scenario 2. Related to our fMRI experiment, there are several considerations regarding experimental design and data analysis that we incorporated from previous studies (Dimoka, 2012; Moshfeghi et al., 2013, 2016).

\section{Task}

Two different search scenarios were given to each participant to complete.

Question-Response (QR) Task: In the first scenario, termed Question-Response (QR), participants were presented with a question for four seconds. Then four possible responses were provided for four seconds while the question remained visible (Figure 1). Participants were not able to make a response until four seconds elapsed of observing the possible responses. We controlled button pressing so that brain activity related to the actual button press would not be entered into the analysis, which considered only these first 8 seconds. After the 8 seconds participants could respond, and for this QR scenario, the experiment then progressed directly to the next trial. Four possible responses were given, and one was always the correct answer, and one was always "need to search". The other two were incorrect answers. The position of the four possible answers was randomised for each trial and response was given by pressing one of the four buttons available on the button box that each participant had in their right hand. This randomisation of button positions prevented effects of motor planning of the button press from systematically influencing results. The order of the questions was randomised for each participant, and they had unlimited time to respond so that they would not be under time pressure to respond.

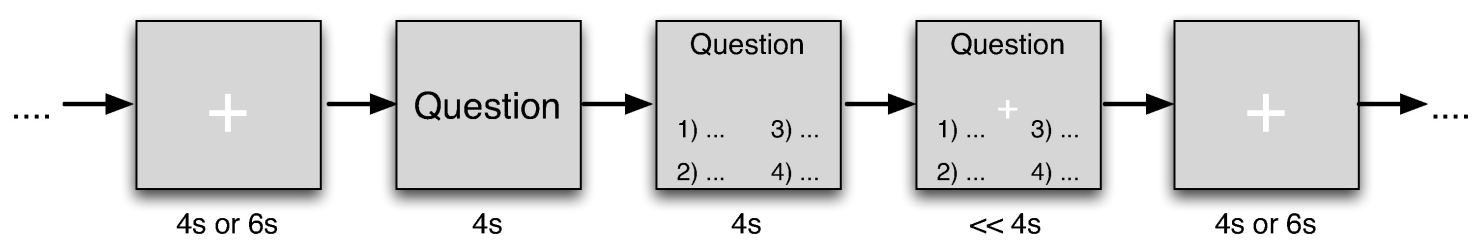

Figure 1. A schematic representation of Scenario 1

Question-Response-Search+ (QRS+) Task: In the second scenario, which we term Question-Response-Search+ (QRS+), participants performed the same task as in the QR scenario except that if the answer "need to search" was provided then they entered an additional stage where they formulated a search query (and submitted it verbally into a noise-cancelling microphone), received a document and read the document with the purpose of evaluating whether it satisfied their information need. They were then given another chance to answer the question, and if they still needed information they were given another opportunity to search. This opportunity to search again was available for two attempts past the first presentation of the question. This extra stage of submitting a query and evaluating 
the received document for possible multiple attempts is denoted by the " +" symbol and distinguishes QRS+ from QRS trials used previously (Moshfeghi et al., 2016).

An illustration of this task is provided in Figure 2. The first part of a trial was identical to Scenario 1 where a question is presented, and multiple possible responses were given. If a participant responded with an answer, the experiment progressed directly to the next trial. However, if the answer "need to search" was provided then this epoch was labelled as IN and participants moved to the next state of the search process. In this next state of query formulation, participants had four seconds to consider what their query was before entering the next state where they were given four seconds to submit their query. Following this, participants viewed a fixation cross on the screen before being presented with a document to read with the purpose of evaluating whether it satisfied their information need. They viewed this document for 16 seconds before returning to the original question and multiple choices. When they responded with an answer to the question (as opposed to the "need to search" option) they were presented with a question of whether they were satisfied with the search and were given four seconds to make this satisfaction judgment. If after seeing the document they still responded with "need to search" they went through another cycle of being presented with another document in response to their query. For any question, there was a limit of three attempts.

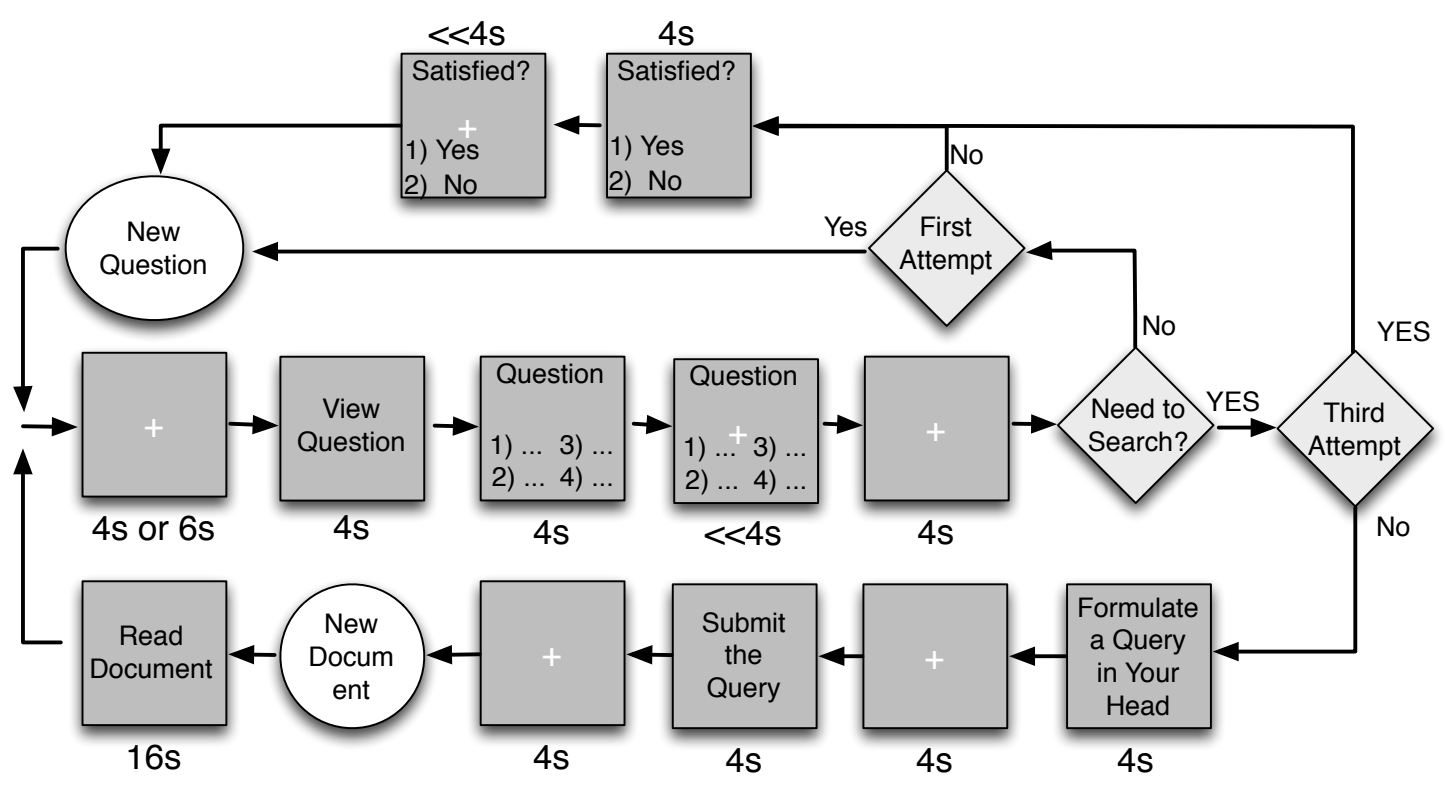

Figure 2. Flowchart of Scenario 2

\section{Question Answering Dataset}

Description of the design of the question answering data set and the documents provided as responses to the queries are provided in (Moshfeghi et al., 2016). Scenario 1 for the QR task had 40 questions covering a variety of topics, 20 of which were easy and 20 were hard questions. Similarly, Scenario 2 for the QRS+ task had 40 questions that were divided into two runs of 20 questions each, with ten easy and ten hard questions. This balance of easy and hard questions was designed so that participants would find themselves in a state of information need for approximately half the trials, and this was evident in the reported 
behavioural results for the QR and QRS tasks (Moshfeghi et al., 2016). A final consideration of question design is that the questions were on topics of general knowledge and did not rely upon particular cognitive skills (e.g. spatial reasoning) or evoke an emotional response. Thus, we can consider the results to reflect IN of general semantic knowledge.

\section{Procedure}

This section outlines the experimental process from start to finish. Ethical permission was obtained from the Ethics Committee of the College of Science and Engineering, University of Glasgow. Participants were recruited from the participant database at the Centre for Cognitive Neuroimaging, University of Glasgow. All participants were informed that the experiment would be around one hour long, including approximately 50 minutes to perform all scans examining information need, and approximately 10 minutes to obtain the anatomical structure of their brain. They were told that they could discontinue participation at any point during the experiment and would still receive their payment of $£ 6 / \mathrm{hr}$ ). Finally, they were asked to sign a consent form. All participants were required to undergo a safety check to confirm that they had no metal items inside or outside of their body, or any other contraindications for scanning.

Before beginning the scan, participants familiarised themselves with the task by performing it on a set of example questions. After successfully completing their training, participants entered the fMRI machine, and the system was adjusted to provide the best comfort and visibility of the screen. While being scanned, each participant first participated in one run of the QR scenario, which contained 40 questions. They then participated in two separate runs of the QRS+ scenario, with each run comprised of 20 questions. It was decided to break the QRS+ scenario into two runs to give the participants a rest during the scanning and to prevent fatigue on the QRS+ task, which consumed more time. After all functional runs were complete, the anatomical data of each participant were obtained.

Upon completion of scanning, participants filled out an exit questionnaire that provided demographic data and qualitative descriptions of their experience during the experiment. They also filled out the Edinburgh handedness questionnaire (Oldfield, 1971), which assesses whether the participant was right-, left- or mixed-handed. Handedness information was obtained since handedness is an indication of the lateralisation of brain function and we wished to ensure that our sample of participants approximated the general population. We conducted a study with this procedure over 15 days from 7 December 2015 to 22 December 2015.

Apparatus: The images were presented using Presentation ${ }^{\circledR}$ software $^{1}$, and projected using an LCD projector onto a translucent screen, while participants watched them in an angled mirror in the MRI scanner.

fMRI Data Acquisition: All fMRI data were collected using a 3T Tim Trio Siemens scanner and 32-channel head coil at the Centre for Cognitive Neuroimaging, University of Glasgow. A functional T2*-weighted MRI run was acquired for the single run of the QR scenario and the two runs of the QRS+ scenario (TR 2000ms; TE 30ms; 32 Slices; $3 \mathrm{~mm}^{3}$ voxel; FOV of 210 , imaging matrix of $70 \times 70$ ). It is worth noting that this $\mathrm{TR}$ timing of $2000 \mathrm{~ms}$ sets the temporal resolution of how frequent brain activity was sampled.

\footnotetext{
${ }^{1}$ Presentation ${ }^{\circledR}$ software (Neurobehavioral Systems, Inc.), http://www.neurobs.com.
} 
At the end of the scanning session, an anatomical scan was performed that involved a high-resolution anatomical scan using a 3D magnetisation prepared rapid acquisition gradient echo (ADNI- MPRAGE) T1-weighted sequence (192 slices; $1 \mathrm{~mm}^{3}$ voxel; Sagittal Slice; $\mathrm{TR}=1900 \mathrm{~ms} ; \mathrm{TE}=2.52 ; 256 \times 256$ image resolution).

Questionnaires: At the end of the experiment, the participants were introduced to an exit questionnaire, which gathered background and demographic information. It also enquired about previous experience with fMRI type user studies as well as participants' general comments for the user study. Finally, it also included questions to ascertain participants' subjective experience of performing the experiment.

Pilot Studies: Before commencing the actual user study, we performed a pilot study using two participants to verify that procedures were working smoothly. Based on this experience and user feedback, we changed a variety of aspects of the experimental paradigm. After the pilot, it was determined that the participants were able to complete the user study without problems, including adequate time to respond to all questions, and that the system was correctly logging participants' interaction data.

\section{fMRI Data Preprocessing}

The fMRI data were preprocessed using Brain Voyager $\mathrm{QX}^{2}$. A standard pipeline of pre-processing of the data was performed for each participant (Goebel, n.d.). This involved slice scan time correction using trilinear interpolation based on information about the TR and the order of slice scanning. Three-dimensional motion correction was performed to detect and correct for small head movements by spatial alignment of all the volumes of a participant to the first volume by rigid body transformations. An exclusion criterion of $3 \mathrm{~mm}$ was used to exclude data where head movement exceeded this amount. Also, linear trends in the data were removed, and high pass filtering with a cutoff of $0.0025 \mathrm{~Hz}$ performed to reduce artefact from low-frequency physiological noise. The functional data were then coregistered with the anatomic data and spatially normalised into the common Talairach space (Talairach \& Tournoux, 1988). Finally, the functional data of each individual underwent spatial smoothing using a Gaussian kernel of $6 \mathrm{~mm}$ to facilitate analysis of group data.

\section{General Linear Model (GLM) Analysis of Scenario 2}

Previous examination of data from the QR task and of a QRS task that did not consider brain activity during the search phase $(\mathrm{QRS}+)$ revealed a set of regions whose activation depended upon information need (Moshfeghi et al., 2016). In the present research, we wished to examine the functional connectivity of these regions during Scenario 1 (QR) and Scenario 2 (QRS+) derived from the same recordings of Moshfeghi et al. (2016). An important factor with the QRS+ data is that the information need scenario is examined over one, two or three attempts depending upon whether the result of the search document satisfies the information need. In order to examine whether there was an interaction between information need and attempt we performed a General Linear Model analysis. Analysis began with a first-level analysis on the data of individual participants using multiple linear regression of the BOLD-response time course in each voxel, using six predictors for the

\footnotetext{
${ }^{2}$ http://www.BrainVoyager.com
} 
different Response Type (respond need to search first attempt, respond with answer first attempt, respond need to search second attempt, respond with answer second attempt, respond need to search third attempt, respond with answer third attempt). To achieve this, for each participant's data a BrainVoyager protocol file (PRT) was derived that represented the onset and duration of the $8 \mathrm{~s}$ total time that the question $(4 \mathrm{~s})$ and question and possible responses $(4 \mathrm{~s})$ were available. Predictors' time courses were adjusted for the hemodynamic response delay by convolution with a hemodynamic response function. Group data were statistically tested with a second-level analysis using a random effects analysis of variance factorial model that used Response Type (IN, no IN) and attempt (first, second, third) as a within-participants factor. To address the issue of multiple statistical comparisons across all voxels, activations are reported using the False Discovery Rate (FDR) at a threshold of $q<0.01$ (Benjamini \& Hochberg, 1995). Using FDR, we control for the number of false positive voxels among the subset of voxels labelled as significant.

\section{Functional Connectivity Analysis}

The results of (Moshfeghi et al., 2016) were produced from contrasting brain activity during IN states to No-IN states and provided us with a set of 8 regions that showed sensitivity to the information need. These results are summarised in Table 1. Some of these clusters were more active when there was an information need, and some were more active when there was not an information need, and this is indicated in the fourth column of Table 1. Likewise, some of the clusters arose from a QR scenario and others from a QRS scenario. To understand better how these different areas interact with one another as a network we examined how the time course of BOLD signals in these select regions was correlated among these areas when participants performed the QR and QRS+ tasks. Note that the QR and QRS segments of the data were identical to data used in Moshfeghi et al. (2016). Such an analysis is related to the connectomics approach to brain imaging (Behrens \& Sporns, 2012; Sporns, 2012) and is known as task-related functional connectivity (DeSalvo, Douw, Takaya, Liu, \& Stufflebeam, 2014; Rissman, Gazzaley, \& D'Esposito, 2004; Vatansever, Menon, Manktelow, Sahakian, \& Stamatakis, 2015). In this approach, the clusters are treated as nodes of a network and the pairwise correlation between nodes as a measure of connectivity between nodes. Consideration of the functional connectivity between the different brain regions further informs our understanding of the related regions as interacting systems rather than just separate brain areas.

To obtain this analysis, as a first step we defined spherical regions of interest (ROI) of radius $4 \mathrm{~mm}$ around the coordinate of peak activity of these regions that showed sensitivity to the information need. This provided 5 ROIs from the QR task condition (caudate head, thalamus, ventral posterior cingulate, caudate body, inferior frontal gyrus) and 3 ROIs from the QRS condition (fusiform gyrus, cuneus, dorsal posterior cingulate). A spherical region centred at the peak of activation, as opposed to the entire cluster of activation, was chosen so that each region would have an equivalent volume. In the next step, we extracted for each participant and run, the entire timecourse of BOLD activation for each spherical ROI. These first steps to extract timecourse activity from volumes of interest were performed using analysis tools provided by Brainvoyager software tools. Following this, we imported the data into Matlab for subsequent network analysis.

The ROI data for each participant contained a single timecourse corresponding to our 
NEUROPSYCHOLOGICAL MODEL OF THE REALISATION OF INFORMATION NEED

Table 1

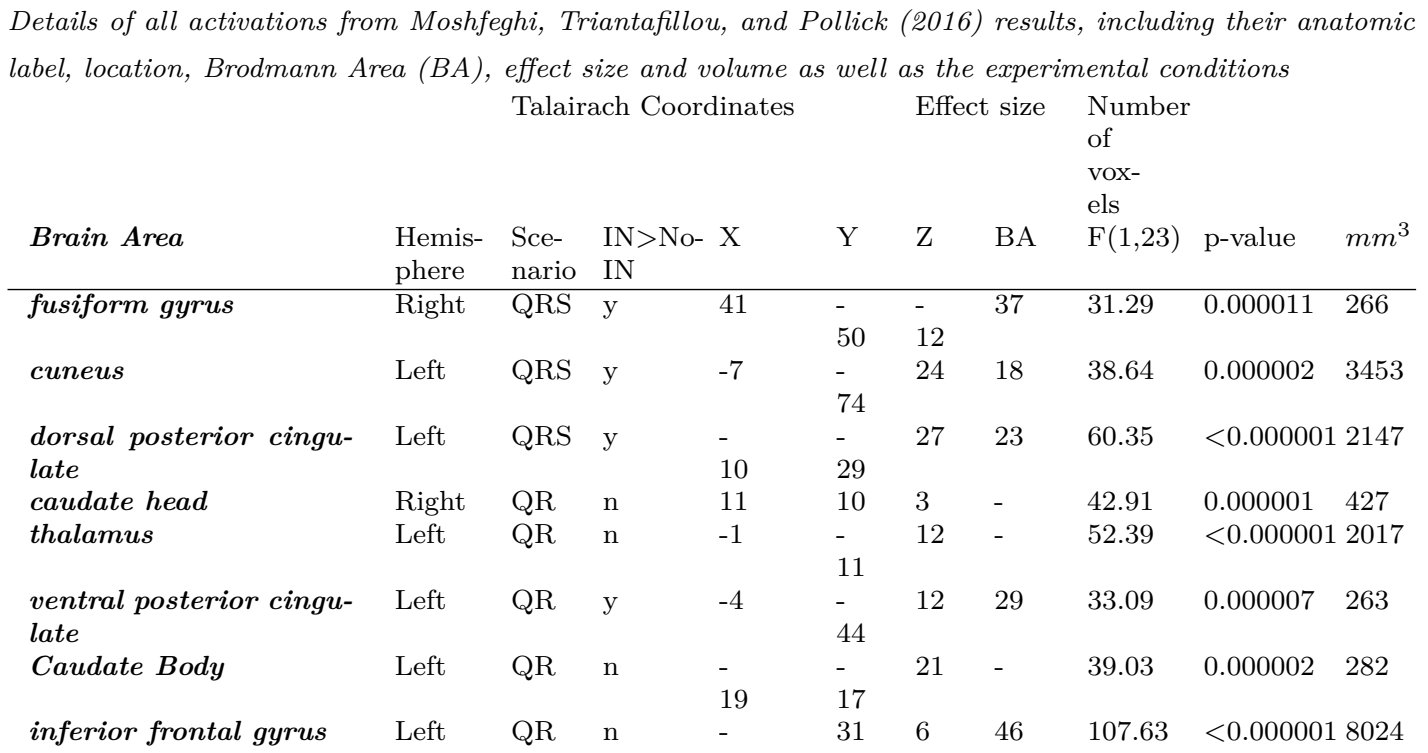

QR, Scenario 1 and two timecourses corresponding to the two runs of our QRS+, Scenario 2. These two runs for Scenario 2 were concatenated into a single file. Subsequent analysis was obtained using tools available in the GraphVar toolbox for Matlab (Kruschwitz, List, Waller, Rubinov, \& Walter, 2015). For each participant and Scenario, a partial-correlation matrix was obtained (Marrelec, Kim, Doyon, \& Horwitz, 2009; Smith et al., 2011; Zalesky, Fornito, $\&$ Bullmore, 2012) and these correlation matrices were entered into a general linear model that examined the functional connectivity between our eight nodes separately for Scenario 1 and Scenario 2. A further analysis was conducted that used a general linear model to inspect for differences between the different networks obtained for Scenario 1 and Scenario 2 .

\section{Results}

\section{General Linear Model Analysis of Scenario 2}

To investigate whether information need had changed throughout Scenario 2 we conducted a random effects general linear model analysis using the factorial design described in General Linear Model (GLM) Analysis of Scenario 2 Section. In particular, we explored the effects of information need, attempt and their interaction across Scenario 2. We were interested in whether there would be an interaction between information need and attempt since this would indicate that information need varied across the different attempts and would complicate interpretation of the network analysis performed over the entire run. However, results showed no interaction effect and no additional regions were found for the main effect of information need. Therefore, for subsequent data analysis in this study, we included all the regions identified by Moshfeghi et al. (2016) into the network analysis and included all attempts at answering the question. We did observe an effect of attempt that we speculate was due to fatigue across attempts. Further, it is possible that failure to find an interac- 
tion between information need and attempt could be due to power issues as there were less second attempts than first attempts and future research might address this important issue.

\section{Functional Connectivity Analysis}

Figure 3 and Figure 4 show the functional connectivity networks of the eight ${ }^{3}$ brain regions of interest (ROI) reported to be sensitive to information need for Scenarios 1 and 2 respectively, as reported by Moshfeghi et al. (2016) and shown in Table 1. The functional connectivity networks are visualised as a graph where the nodes represent the brain regions under consideration, and the links represent the fact that statistically significant correlation values (see Functional Connectivity Analysis Section) were obtained between the nodes. The thicker links indicate larger correlation coefficients. The structure of these two networks addresses RQ1. The four nodes that are red correspond to the ROIs where greater activity was found when there was no information need (No-IN), and the four nodes that are yellow correspond to the ROIs where greater activity was found for information need (IN). For evaluation of these networks, we used a criterion of $p<0.05$ corrected for multiple comparisons using Bonferroni correction to obtain a criterion of $(p<0.00078)$. Exploring the relation between IN and No-IN regions show that in both scenarios there are links between the IN (yellow) and No-IN (red) regions, indicating that these areas are inter-related to one another, rather than separate systems. This addresses RQ2.

L
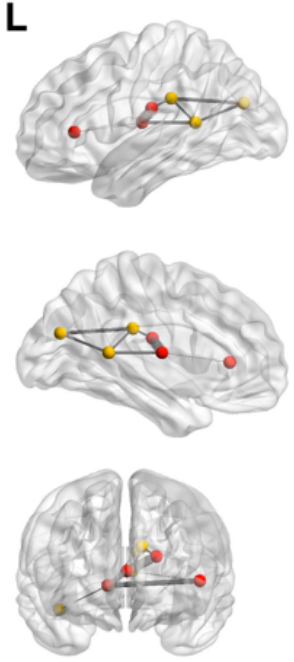
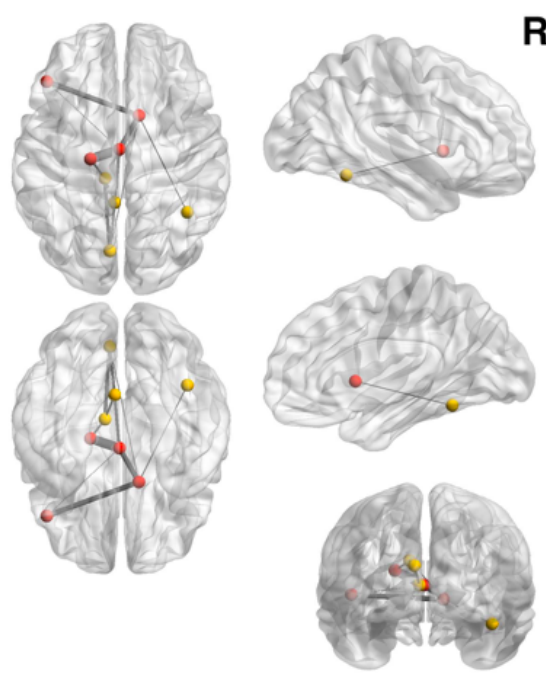

$\mathbf{R}$

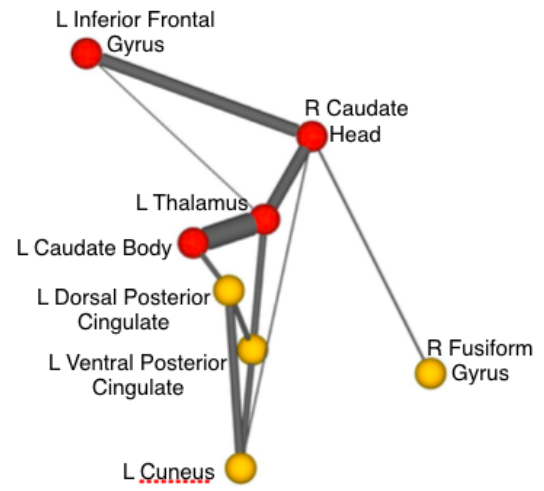

Figure 3. Results of Scenario 1. The functional connectivity network of the eight nodes explored is overlaid in their appropriate brain location in the left panel and shown schematically in the right panel. The four nodes that are red correspond to the ROIs where greater activity was found when there was no information need (No-IN), and the four nodes that are yellow correspond to the ROIs where greater activity was found for information need (IN). The lines correspond to links that had statistically significant correlation coefficients and thicker lines between the nodes indicate larger correlation coefficients.

\footnotetext{
${ }^{3}$ Although examination of a small number of nodes such as eight precludes computation of more sophisticated connectivity metrics (Rubinov \& Sporns, 2010), it is still possible to statistically compare two networks, and we can also expect trends of connectivity to be qualitatively visible.
} 

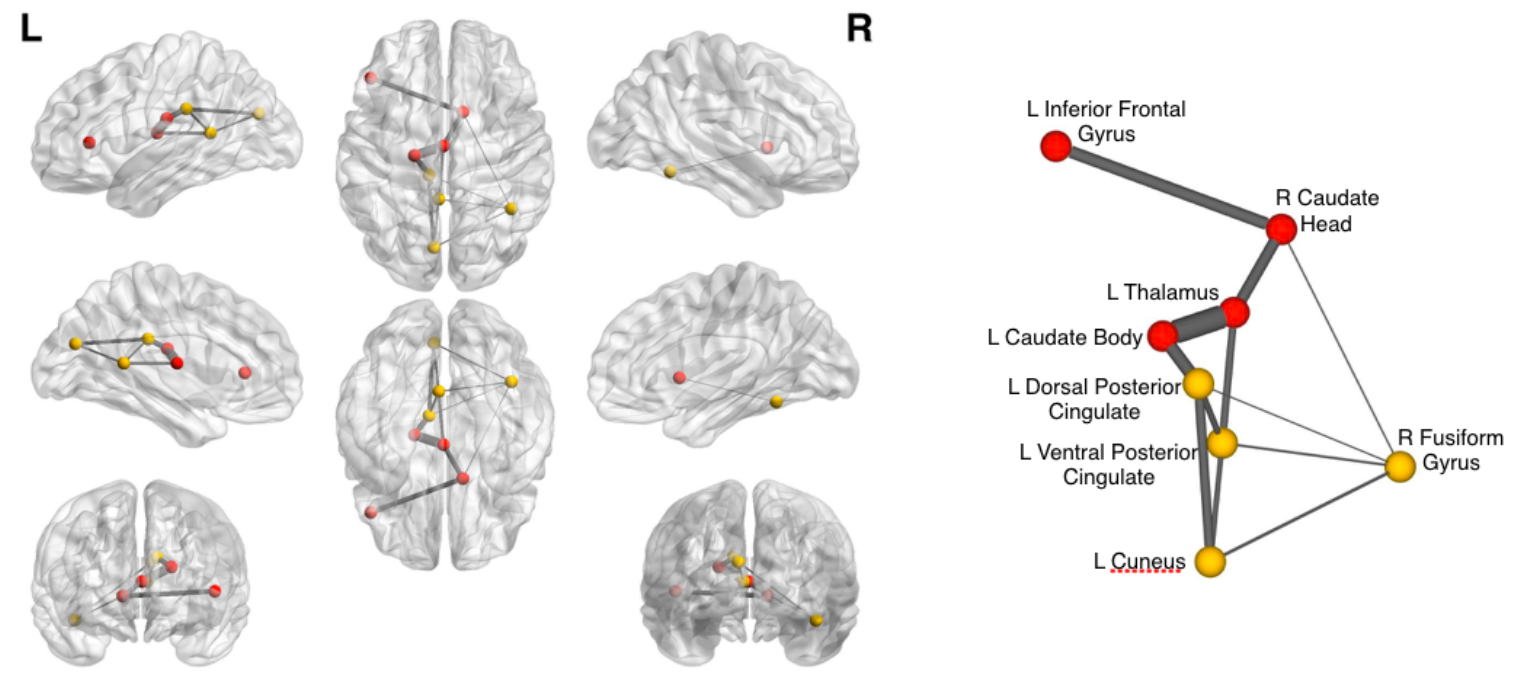

Figure 4. Results of Scenario 2. The functional connectivity network of the eight nodes explored is overlaid in their appropriate brain location in the left panel and shown schematically in the right panel. The four nodes that are red correspond to the ROIs where greater activity was found when there was no information need (No-IN), and the four nodes that are yellow correspond to the ROIs where greater activity was found for information need (IN). The lines correspond to links that had statistically significant correlation coefficients and thicker lines between the nodes indicate larger correlation coefficients.

\section{Network Comparison}

Visual inspection of Figures 3 and 4 reveals numerous similarities between the two networks. These similarities are broadly consistent with the fact that in both scenarios approximately half the questions would be given a response retrieved from memory. Therefore, it is expected that the brain connectivity would be similar for such items if they occurred either in Scenario 1 or Scenario 2.

Comparison of the networks of Scenario 1 and Scenario 2 revealed significant differences between the networks ( $p<0.05$, uncorrected for multiple comparisons). The first difference was an increased functional connectivity between dorsal posterior cingulate and caudate body for Scenario 2 in comparison to Scenario 1. The second difference was a connection between ventral posterior cingulate and fusiform gyrus in Scenario 2, where such a connection was not observed in Scenario 1. These findings further underscore the role of the posterior cingulate in differentiating between states of IN without search (Scenario 1) and with search (Scenario 2).

Another difference between scenarios was the connectivity of the fusiform gyrus between the scenarios. In Scenario 1 it showed connectivity with only the caudate head, but in Scenario 2 this connectivity increased to include connections with cuneus as well as dorsal and ventral posterior cingulate. This greater connectivity of the fusiform gyrus in Scenario 2 suggests that this region relates to integrating high-level visual properties when participants are in a state of information need. 


\section{Information Need Realisation Model}

Comparing and contrasting the network analyses of the two scenarios and their related tasks leads to a view of how the eight regions sensitive to information need might work together as an integrated system. We propose that breaking down these regions into three inter-relating components provides a useful model of the processes involved. These include

- Component 1: a successful memory retrieval component (left thalamus, left caudate Body, right caudate head, left inferior frontal gyrus),

- Component 2: an information flow regulation component (left cuneus, left dorsal posterior cingulate, left ventral posterior cingulate), and

- Component 3: a high-level perception component (right fusiform gyrus).

Below we discuss each component in detail.

\section{Component 1 (Successful Memory Retrieval):}

The successful memory retrieval component includes left thalamus, left caudate body, right head of caudate, and the left inferior frontal gyrus which is also known as the dorsolateral prefrontal cortex. These four regions arose from the information need sensitive regions that showed higher activation for the No-IN than the IN condition. In the results of Moshfeghi et al. (2016) these regions were identified for the QR task but not the QRS task, raising the issue of whether they were not as active for the QRS task when there was not an information need. However, the connectivity of these regions was nearly identical in both scenarios, and this is compatible with, regardless of the scenario, the involvement of this set of regions in correct memory retrieval. Moreover, these four regions, as evidenced by the strength of their correlation (represented by the thickness of the lines connecting them), had the greatest magnitude of functional connectivity for both scenarios in a pattern that linked all regions along a single pathway. These functional connectivity results are consistent with the finding that these regions are known to have anatomical connectivity (Draganski et al., 2008). In addition, the function of these regions include processes of memory retrieval (A. S. Mitchell \& Chakraborty, 2013), working memory (Barbey, Koenigs, \& Grafman, 2013), and decision making (Ding \& Gold, 2010). These functions are consistent with expectations of what a pathway for successful memory retrieval would include and support why the component would show similar functional connectivity for both scenarios.

An additional comment regarding the dorsolateral prefrontal cortex area of the inferior frontal gyrus is that this region has been identified as an area involved in factual searches (Mostafa, Carrasco, Foster, \& Giovenallo, 2015). However, possibly more important in relating the results in left dorsolateral prefrontal cortex to items with successful memory retrieval is that our findings are also consistent with what is known about lateralisation of the dorsolateral prefrontal cortex, where the right hemisphere is involved in coarse processing of language that can be related to making inferences about discourse (Beeman, 1993), and the left hemisphere is more involved in fine coding of semantic features and appears active for semantic items drawn into consciousness for further processing (R. L. Mitchell, Vidaki, \& Lavidor, 2016), which is consistent with an answer drawn into consciousness. While our task was language based, the activity found in left dorsolateral prefrontal cortex 
might not necessarily be language-based as it has been shown that some regions of left dorsolateral prefrontal cortex activate independent of response or input modality (Heekeren, Marrett, Ruff, Bandettini, \& Ungerleider, 2006). Consistent with this are data indicating that damage to left dorsolateral prefrontal cortex is related to general intelligence Barbey et al., 2013. These views of the dorsolateral prefrontal cortex also help to put our findings in the context of metamemory research that has examined the role of the dorsolateral prefrontal cortex in FOK judgments. These results, similar to ours on the contrast of IN versus No-IN show greater activation in dorsolateral prefrontal cortex when participants provide a strong FOK than when they report a weak FOK (Chua, Schacter, \& Sperling, 2009).

\section{Component 2 (Information Flow Regulation):}

The information flow regulation component involves the cuneus and the dorsal and ventral posterior cingulate. The cuneus, although more often related to visual processing, is also known to be involved in higher level cognition such as autobiographical memory (Addis, McIntosh, Moscovitch, Crawley, \& McAndrews, 2004). The posterior cingulate is known to be a metabolically active area that uses substantial energy and serves as a hub that regulates cognitive activity (van den Heuvel \& Sporns, 2013). It is especially involved in regulating brain resources between engaging in internal or external processes, and differences between activation of dorsal and ventral posterior cingulate have been related to theories of the posterior cingulate and its special role in brain function, specifically the Arousal, Balance and Breadth of Attention (ABBA) model (Leech \& Sharp, 2014). The ABBA model holds that activation in ventral posterior cingulate is consistent with a narrow internal focus while activation in dorsal posterior cingulate is consistent with a broad external focus. In relation to information need, this distinction between narrow internal and broad external focus was evident in the results of Moshfeghi and colleagues (Moshfeghi et al., 2016). The present functional connectivity results show that both dorsal and ventral posterior cingulate increase their connectivity during Scenario 2. While these connectivity results between scenarios are equivocal on the differentiation of dorsal versus ventral posterior cingulate they are consistent with the view of posterior cingulate as a "hub" region that is densely

connected with other parts of the brain and the idea of recruitment of additional resources in Scenario 2 when external information and broad focus is required.

The regions involved in Component 2 have also been related to metamemory research investigating FOK in a study by Elman, Klostermann, Marian, Verstaen, and Shimamura (2012) exploring neural correlates of metacognitive monitoring during retrieval of semantic and episodic memories. For semantic memories, they found greater activation in left ventral posterior cingulate when they contrasted brain activity for strong FOK items to weak FOK items which is consistent with our proposed role for the ventral posterior cingulate. For the contrast of weak FOK to strong FOK for semantic memories, they reported activation in dorsal posterior parietal cortex, which is not entirely consistent with our reporting of the cuneus, though the examination of FOK strength in the context of information need deserves future study. 
NEUROPSYCHOLOGICAL MODEL OF THE REALISATION OF INFORMATION

NEED

\section{Component 3 (High-Level Perception):}

The fusiform has been implicated in a variety of domains. One established view of the fusiform area is that the left hemisphere (language dominant) is involved in reading and the transformation of writing symbols into phonetic and semantic codes (McCandliss, Cohen, \& Dehaene, 2003), while the right hemisphere is involved in the perception and categorization of visual objects such as faces (Kanwisher \& Yovel, 2006; Tarr \& Gauthier, 2000). It has also been implicated in the processing of emotions (Domes et al., 2010) as well as cognitive and perceptual aspects of language processing (Kuperberg et al., 2000; Lee, Truy, Mamou, Sappey-Marinier, \& Giraud, 2007) . While the current findings do not neatly fit into this literature what these views have in common is that, although specialized processing is lateralized, the fusiform can be thought of as a high-level visual area that serves cognitive representations of visual input. In both scenarios the input to participants was visual, and thus the results are consistent with this general view. The current results are not consistent with the lateralization hypothesis since we would expect the left, not the right fusiform to be active since the visual input was written language. However, it is possible that lateralization is not complete and some language processing particular to the current task resides in the right hemisphere.

Consistent with this idea that fusiform is related to high level visual representations is the finding that it has been related to conscious representations of aspects of viewed objects (Vandenbulcke, Peeters, Fannes, \& Vandenberghe, 2006). A mechanism proposed for this role of the fusiform is that it is involved not only in the feedforward processing of visual information in a bottom-up fashion but also in processing that includes top-down feedback from higher brain areas that aid in the interpretation of visual stimuli. While this account was derived to explain visual object processing and not for processing written text, the general idea of the fusiform being involved in top-down processing is entirely consistent with our results. For example, in Scenario 2 when individuals had to perform a search task was when we saw connectivity between the right fusiform and the other regions that made up our information flow regulation component.

It would thus seem parsimonious to consider the right fusiform area to be a brain region that represents complex visual properties and receives top-down influences from higher brain regions. We can speculate that these processes could even possibly include representation of visual properties extracted from memory. While the current results identifying right fusiform arise from a visual task, it might be expected that for a listening task another brain region in the auditory processing stream might serve an analogous function.

\section{Model Summary}

It is useful to summarise the proposed components in relation to their role in the realisation of information need. Component 2 involves the regulation of information flow and includes those regions proposed by Moshfeghi et al. (2016) to be neural correlates of realising information need. In particular, it was argued that the brain activity in dorsal posterior cingulate reflects broad external search as would be expected when a person realises that desired information is not available internally and orients the recruitment of neural resources towards external search. Thus, we can consider this regulation of information flow demonstrated by Component 2 of our model to be most closely related to the brain 
state of realising information need. Moreover, the present network analysis suggests that this state is part of a dynamic process that balances memory access (Component 1) and perceptual processing (Component 2).

\section{Discussion and Conclusion}

How information need occurs and what processes form the basis of its existence is a critical question in IR. Information need refers to a complex concept: at the very initial state of the phenomenon (i.e. at a visceral level), even the searcher may not be aware of its existence. Answering this question has proven problematic given its subjective nature and the limited means to experimentally probe such tacit states of experience. However, the recent rapid advance of neurophysiological measures such as functional Magnetic Resonance Imaging (fMRI) has provided means to quantify the neural states related to Information Need and thus provide insight into how Information Need comes about.

In this paper, using an fMRI study, we measured the brain activity of twenty-four participants while they performed a Question Answering (Q/A) Task, where the questions were carefully selected and developed from TREC-8 and TREC 2001 Q/A Track. We collected new data of brain activity while participants formulated and stated a realisation of IN, focusing on the distributed network of eight brain regions sensitive to information need found in our previous study (Moshfeghi et al., 2016). These data were analysed by examining task-based functional connectivity of the eight brain regions in two different scenarios. In the first scenario individuals received a query and merely stated that they needed to search, and in the second scenario a dynamic search process was incorporated that allowed participants up to three attempts to either answer a question or submit a query to gather additional information.

Examination of task-based functional connectivity among these regions over different scenarios revealed evidence of a consistent network structure across scenarios (addressing RQ1), and that IN nodes of the network are connected to No-IN nodes in the network (addressing RQ2). Although there were many similarities between the networks found for Scenario 1 and Scenario 2, differences were found in the patterns of functional connectivity. For Scenario 2 we found two linkages between dorsal and ventral posterior cingulate to other regions that had statistically significant greater functional connectivity during Scenario 2. Also, there was a qualitative increase in the connectivity of the fusiform gyrus to other regions during Scenario 2. These differences address RQ3 and within the context of the model proposed reveal that for Scenario 2 there was greater connectivity between the Information Flow component with the other two components as well as an increase in functional connectivity to high-level visual information.

These results add to our understanding of information need obtained from (Moshfeghi et al., 2016) and contribute to a more holistic view of how IN is reflected in brain activity. In this previous study, a GLM analysis revealed different brain regions for the different scenarios. However, by examining functional connectivity over all these regions for both scenarios, we were able to find a more holistic view of how these regions interact that goes beyond what was revealed by the GLM. Indeed, although across the two scenarios, this

previous study revealed eight areas, four of which were more active for the Information Need state and four which were more active for the No-INformation Need state, we did not 
have an idea of the interrelationship between the regions. Moreover, some of the regions found were not consistently identified across experimental conditions.

The present task-based functional connectivity analysis across the two scenarios helps to provide a unified, holistic view of how these regions interact, leading us to propose a neuropsychological model of the realisation of IN. Our model consists of three components: (i) a successful memory retrieval component, (ii) an information flow regulation component, and (iii) a high-level perception component. The first component includes links connecting left thalamus, left caudate body, right caudate head, and left inferior frontal gyrus, involving when participants provide an answer to a given query, i.e. successful memory retrieval. The second component connects to the other two components and includes left cuneus, left dorsal posterior cingulate, and left ventral posterior cingulate, and involves the switching between internal and external information. Finally, the third component includes right fusiform gyrus which is thought to be involved in complex representations for visual cognition.

\section{Limitations and Future Directions}

One form of limitations in the current work arises from the issue that Information Need is a complex mental phenomenon that involves other perceptual and cognitive processes. Moreover, there is scarce research into the neural processes that underlie Information Need, and thus it is difficult to position our findings within a larger context. While drawing connections with findings from metacognition help, there is still much work to be done in building literature on this topic. The intrinsic problem is that due to the complexity of the problem of Information Need, it is difficult to design experiments that isolate particular aspects of the problem. We hope that our framework of three components helps to guide future study that must address these issues. Given the complexity of Information Need, we also need to address the issue of how our current findings will generalize to different tasks or different populations. While we would expect the components of our model to generalize, it can be hypothesized that tasks involving a need of spatial or episodic information might invoke other specialized brain regions. Similarly, particular groups of individuals might systematically vary from typical in how information need is realized due to experience (e.g. domain experts), development (e.g. autism spectrum disorder), disease (e.g. major depression disorder) or personality (risk averse or not) . Future work would profit from an ability to understand Information Need at the level of the individual to provide a personalised analysis of brain activity. An additional limitation that needs to be addressed is that due to the restrictive nature of acquiring brain data in an experimental context it was necessary to control passage of participants through the various stages of information need and this might have unintended consequences. For example, participants were given sixteen seconds to assess a document, and while it assured every document could be comprehended, if a participant finished sooner they had to wait, which might have resulted in boredom or mind wandering that would effectively produce unwanted activity in the brain data. Related to this is that while the experiment was designed so that the effect of button pressing was minimised, holding the motor plan for a button press over longer periods might introduce brain activity that is not essential for illuminating the core issues of information need. Future study designs might develop that allow a more naturalistic and self-paced progression through experimental conditions.

As Neuro-Information Science is a young field, it is also important to reflect upon 
what properties are involved in the match between instrument capabilities, measurements, IR constructs and neural models. Particularly how these considerations might inform future work that goes past the limits of these present exploratory experiments and the neural processing model proposed. To illustrate this point we can consider that the nodes for our functional connectivity network analysis came from a General Linear Model (GLM) analysis of a contrast of fMRI BOLD signal on trials where participants indicated there was an IN, versus when there was no IN (Moshfeghi et al., 2016). This analysis was based on an assumption of cognitive subtraction between the two states, and it has been argued that cognitive subtraction in tandem with a GLM analysis risks missing brain areas that involve nonlinear interactions between neural components (Friston et al., 1996). Such nonlinearities are evident in our own data, for example, that the contrast of IN versus No-IN trials yielded differences between the two scenarios shows that scenario interacts with IN.

Another assumption is that IN has implicitly been treated as a continuous variable, rather than as a dichotomous variable. Although treating IN as a continuous variable might be appropriate, as gradations in IN might represent confidence or degree of certainty, in the future we aim to look closer at these properties of our conceptual models to further develop the proposed neural model. For example, considering the neural model, possibly the successful memory retrieval component is dichotomous, and the flow regulation component acts in a continuous fashion or possibly both act in a dichotomous fashion, and it is from their interplay that a continuous value of IN is obtained. Possibly the entire system is dichotomous, and continuity arises as an epiphenomenon of the measurement and data preprocessing stages. One potential way forward to explore this would be to use multivariate techniques that are better suited to deal with data nonlinearities. Another approach, following the lead of Kikyo et al. (2002) in their study of metacognition would be to avoid the assumptions inherent in cognitive subtraction and adopt a parametric approach for our experimental design. In a parametric approach, psychological judgments of the level of information need could be used to analyse the brain data. These studies can be guided by the model proposed in this paper.

In conclusion, our IN realisation model arose from an interpretation of the current data, a previous examination of the neural substrate involved in Information Need (Moshfeghi et al., 2016), and the broader brain imaging and cognitive neuroscience literature. In particular, our findings show a close relationship with neurocognitive studies of FOK in metacognition, which reinforces the similarities of IN and FOK at a behavioural level. Future studies could explore this connection in greater depth and expand to other metacognitive phenomena such as the tip of the tongue experience and judgments of learning. This model is the first of its kind to be based on a systems neuroscience understanding of Information Need and is driven by the unique requirements of understanding Information Need, where an organism must balance what is known internally with what can be gathered from the external environment. The model helps to view the fundamental process of Information Need within the context of inter-relating subnetworks that act to realise the assorted psychological processes subsumed by Information Need. We believe our study and our neuropsychological model of the realisation of IN constitute an important step in unravelling the nature of information need and will have a great impact in directing future works into how to better satisfy IN. 
NEUROPSYCHOLOGICAL MODEL OF THE REALISATION OF INFORMATION

NEED

\section{References}

Addis, D. R., McIntosh, A. R., Moscovitch, M., Crawley, A. P., \& McAndrews, M. P. (2004). Characterizing spatial and temporal features of autobiographical memory retrieval networks: a partial least squares approach. Neuroimage, 23(4), 1460-1471.

Allegretti, M., Moshfeghi, Y., Hadjigeorgieva, M., Pollick, F. E., Jose, J. M., \& Pasi, G. (2015). When relevance judgement is happening?: an eeg-based study. In Sigir '15. New York, NY, USA: ACM.

Barbey, A. K., Koenigs, M., \& Grafman, J. (2013). Dorsolateral prefrontal contributions to human working memory. Cortex, 49(5), 1195-1205.

Bates, M. J. (1998). Indexing and access for digital libraries and the internet: human, database, and domain factors. JASIST, 49(13), 1185-1205.

Beeman, M. (1993, January). Semantic Processing in the Right-Hemisphere May Contribute to Drawing Inferences From Discourse. Brain and Language, 44 (1), 80-120.

Behrens, T. E. \& Sporns, O. (2012). Human connectomics. Current Opinion in Neurobiology, 22(1), 144-153. Neurotechnology. doi:http://dx.doi.org/10.1016/j.conb.2011.08.005

Belkin, N. J., Oddy, R. N., \& Brooks, H. M. (1982a). ASK for information retrieval: Part I. Background and theory. Journal of Documentation, 38(2), 61-71.

Belkin, N. J., Oddy, R. N., \& Brooks, H. M. (1982b). ASK for information retrieval: Part II. Results of a design study. Journal of documentation, 38(3), 145-164.

Benjamini, Y. \& Hochberg, Y. (1995). Controlling the false discovery rate: a practical and powerful approach to multiple testing. Journal of the Royal Statistical Society. Series $B$ (Methodological), 289-300.

Borgman, C. L. (2000). From gutenberg to the global information infrastructure: access to information in the networked world. Mit Press.

Chua, E. F., Pergolizzi, D., \& Weintraub, R. R. (2014, February). The Cognitive Neuroscience of Metamemory Monitoring: Understanding Metamemory Processes, Subjective Levels Expressed, and Metacognitive Accuracy. In The cognitive neuroscience of metacognition (pp. 267-291). Berlin, Heidelberg: Springer Berlin Heidelberg.

Chua, E. F., Schacter, D. L., \& Sperling, R. A. (2009, September). Neural Correlates of Metamemory: A Comparison of Feeling-of-Knowing and Retrospective Confidence Judgments. Journal of Cognitive Neuroscience, 21 (9), 1751-1765.

Cole, C. (2011). A theory of information need for information retrieval that connects information to knowledge. JASIST, 62 (7), 1216-1231.

Cole, C., Julien, C.-A., \& Leide, J. E. (2010). An associative index model for hypertext internet search based on vannevar bush's memex machine: an exploratory case study. Information Research, 15(3), 15-3.

DeSalvo, M. N., Douw, L., Takaya, S., Liu, H., \& Stufflebeam, S. M. (2014). Task-dependent reorganization of functional connectivity networks during visual semantic decision making. Brain and behavior, 4(6), 877-885.

Dimoka, A. (2012). How to conduct a functional magnetic resonance (fmri) study in social science research. MIS Quarterly, 36(3), 811-840.

Ding, L. \& Gold, J. I. (2010). Caudate encodes multiple computations for perceptual decisions. The Journal of Neuroscience, 30(47), 15747-15759. 
Domes, G., Schulze, L., Boettger, M., Grossmann, A., Hauenstein, K., Wirtz, P. H., ... Herpertz, S. C. (2010, May). The Neural Correlates of Sex Differences in Emotional Reactivity and Emotion Regulation. Human Brain Mapping, 31 (5), 758-769.

Draganski, B., Kherif, F., Klöppel, S., Cook, P. A., Alexander, D. C., Parker, G. J., ... Frackowiak, R. S. (2008). Evidence for segregated and integrative connectivity patterns in the human basal ganglia. The Journal of Neuroscience, 28(28), 7143-7152.

Elman, J. A., Klostermann, E. C., Marian, D. E., Verstaen, A., \& Shimamura, A. P. (2012, May). Neural correlates of metacognitive monitoring during episodic and semantic retrieval. Cognitive, Affective, \& Behavioral Neuroscience, 12(3), 599-609.

Eugster, M. J. A., Ruotsalo, T., Spapé, M. M., Barral, O., Ravaja, N., Jacucci, G., \& Kaski, S. (2016, November). Natural brain-information interfaces: Recommending information by relevance inferred from human brain signals. Nature Publishing Group, $1-10$.

Eugster, M. J., Ruotsalo, T., Spapé, M. M., Kosunen, I., Barral, O., Ravaja, N., .. Kaski, S. (2014). Predicting term-relevance from brain signals. In Sigir'14 (pp. 425-434). ACM.

Flavell, J. H. (1979). Metacognition and cognitive monitoring: A new area of cognitivedevelopmental inquiry. American Psychologist, 34(10), 906-911.

Fleming, S. M. \& Dolan, R. J. (2012, April). The neural basis of metacognitive ability. Philosophical transactions of the Royal Society of London. Series B, Biological sciences, 367(1594), 1338-1349.

Friston, K. J. (2011). Functional and effective connectivity: a review. Brain connectivity, $1(1), 13-36$.

Friston, K. J., Price, C., Fletcher, P., Moore, C., Frackowiak, R., \& Dolan, R. J. (1996). The trouble with cognitive subtraction. Neuroimage, 4(2), 97-104.

Goebel, R. (n.d.). Brainvoyager qx, vers.2.1, brain innovation b.v. Maastricht, Netherlands.

Gwizdka, J., Hosseini, R., Cole, M., \& Wang, S. (2017, August). Temporal dynamics of eyetracking and EEG during reading and relevance decisions. Journal of the Association for Information Science and Technology, 68(10), 2299-2312.

Gwizdka, J. \& Mostafa, J. (2016, January). NeuroIR 2015 - SIGIR 2015 Workshop on Neuro-Physiological Methods in IR Research, 1-6.

Heekeren, H. R., Marrett, S., Ruff, D. A., Bandettini, P. A., \& Ungerleider, L. G. (2006). Involvement of human left dorsolateral prefrontal cortex in perceptual decision making is independent of response modality. Proceedings of the National Academy of Sciences, $103(26), 10023-10028$.

Hjørland, B. (2010). The foundation of the concept of relevance. JASIST, 61 (2), 217-237.

Ingwersen, P. (1996). Cognitive perspectives of information retrieval interaction: elements of a cognitive ir theory. Journal of documentation, 52(1), 3-50.

Ingwersen, P. \& Järvelin, K. (2006). The turn: integration of information seeking and retrieval in context. Springer, 2005. xiv, 448 S. ISBN 1-4020-3850-X.

Kanwisher, N. \& Yovel, G. (2006). The fusiform face area: a cortical region specialized for the perception of faces. Philosophical transactions of the Royal Society of London. Series B, Biological sciences, 361 (1476), 2109-2128. 
Kauppi, J. ., Kandemir, M., Saarinen, V. ., Hirvenkari, L., Parkkonen, L., Klami, A., ... Kaski, S. (2015). Towards brain-activity-controlled information retrieval: Decoding image relevance from MEG signals. NeuroImage, in press.

Kelly, D. \& Fu, X. (2007, January). Eliciting better information need descriptions from users of information search systems. Inf. Process. Manage. 43(1), 30-46.

Kikyo, H., Ohki, K., \& Miyashita, Y. (2002, September). Neural correlates for feeling-ofknowing: an fMRI parametric analysis. Neuron, 36(1), 177-186.

Kruschwitz, J., List, D., Waller, L., Rubinov, M., \& Walter, H. (2015). Graphvar: a userfriendly toolbox for comprehensive graph analyses of functional brain connectivity. Journal of Neuroscience Methods, 245, 107-115. doi:http://dx.doi.org/10.1016/j. jneumeth.2015.02.021

Kuhlthau, C. C. (1993). A principle of uncertainty for information seeking. Journal of Documentation, 49(4), 339-355.

Kuperberg, G. R., McGuire, P. K., Bullmore, E. T., Brammer, M. J., Rabe-Hesketh, S., Wright, I. C., ... David, A. S. (2000, March). Common and distinct neural substrates for pragmatic, semantic, and syntactic processing of spoken sentences: an fMRI study. Journal of Cognitive Neuroscience, 12(2), 321-341.

Lee, H.-J., Truy, E., Mamou, G., Sappey-Marinier, D., \& Giraud, A.-L. (2007, November). Visual speech circuits in profound acquired deafness: a possible role for latent multimodal connectivity. Brain, 130(Pt 11), 2929-2941.

Leech, R. \& Sharp, D. J. (2014). The role of the posterior cingulate cortex in cognition and disease. Brain, 137(1), 12-32.

Maril, A., Simons, J. S., Mitchell, J. P., Schwartz, B. L., \& Schacter, D. L. (2003, April). Feeling-of-knowing in episodic memory: an event-related fMRI study. NeuroImage, 18(4), 827-836.

Marrelec, G., Kim, J., Doyon, J., \& Horwitz, B. (2009). Large-scale neural model validation of partial correlation analysis for effective connectivity investigation in functional mri. Human Brain Mapping, 30(3), 941-950. doi:10.1002/hbm.20555

McCandliss, B. D., Cohen, L., \& Dehaene, S. (2003, July). The visual word form area: expertise for reading in the fusiform gyrus. Trends in Cognitive Sciences, 7(7), 293299.

Mitchell, A. S. \& Chakraborty, S. (2013). What does the mediodorsal thalamus do? Frontiers in systems neuroscience, $\%$.

Mitchell, R. L., Vidaki, K., \& Lavidor, M. (2016, October). The role of left and right dorsolateral prefrontal cortex in semantic processing - A transcranial direct current stimulation study. Neuropsychologia, 91(100), 480-489.

Moshfeghi, Y., Pinto, L. R., Pollick, F. E., \& Jose, J. M. (2013). Understanding relevance: an fmri study. In Ecir (pp. 14-25).

Moshfeghi, Y., Triantafillou, P., \& Pollick, F. E. (2016). Understanding information need: an fmri study. In Proceedings of the 39th international acm sigir conference on research and development in information retrieval (pp. 335-344). SIGIR '16. Pisa, Italy: ACM. doi:10.1145/2911451.2911534

Mostafa, J., Carrasco, V., Foster, C., \& Giovenallo, K. (2015). Identifying Neurological Patterns Associated with Information Seeking: A Pilot fMRI Study. In Information systems and neuroscience (pp. 167-173). Cham: Springer, Cham. 
Nelson, T. O. \& Narens, L. (1990, January). Metamemory: A Theoretical Framework and New Findings. In Psychology of learning and motivation - advances in research and theory (pp. 125-173). Elsevier.

Oldfield, R. C. (1971). The assessment and analysis of handedness: the edinburgh inventory. Neuropsychologia, 9(1), 97-113.

Rissman, J., Gazzaley, A., \& D'Esposito, M. (2004). Measuring functional connectivity during distinct stages of a cognitive task. NeuroImage, 23(2), 752-763. doi:https: //doi.org/10.1016/j.neuroimage.2004.06.035

Rubinov, M. \& Sporns, O. (2010). Complex network measures of brain connectivity: uses and interpretations. NeuroImage, 52(3), 1059-1069. Computational Models of the Brain. doi:https://doi.org/10.1016/j.neuroimage.2009.10.003

Smith, S. M., Miller, K. L., Salimi-Khorshidi, G., Webster, M., Beckmann, C. F., Nichols, T. E., ... Woolrich, M. W. (2011). Network modelling methods for ffmri\}. NeuroImage, 54(2), 875-891. doi:https://doi.org/10.1016/j.neuroimage.2010.08.063

Sporns, O. (2012). From simple graphs to the connectome: networks in neuroimaging. NeuroImage, 62(2), 881-886. 20 YEARS OF fMRI. doi:http://dx.doi.org/10.1016/j. neuroimage.2011.08.085

Talairach, J. \& Tournoux, P. (1988). Co-planar stereotaxic atlas of the human brain. Thieme New York:

Tarr, M. J. \& Gauthier, I. (2000, August). FFA: a flexible fusiform area for subordinate-level visual processing automatized by expertise. Nature Neuroscience, 3(8), 764-769.

Taylor, R. S. (1968). Question-negotiation and information seeking in libraries. College 86 research libraries, 29(3), 178-194.

van den Heuvel, M. P. \& Sporns, O. (2013). Network hubs in the human brain. Trends in cognitive sciences, $17(12), 683-696$.

van Rijsbergen, C. (1986). (invited paper) A new theoretical framework for information retrieval. In Sigir (pp. 194-200). ACM.

Vandenbulcke, M., Peeters, R., Fannes, K., \& Vandenberghe, R. (2006, July). Knowledge of visual attributes in the right hemisphere. Nature Neuroscience, 9(7), 964-970.

Vatansever, D., Menon, D., Manktelow, A., Sahakian, B., \& Stamatakis, E. (2015). Default mode network connectivity during task execution. NeuroImage, 122, 96-104. doi:http: //dx.doi.org/10.1016/j.neuroimage.2015.07.053

White, R. W. (2004). Implicit Feedback for Interactive Information Retrieval (Doctoral dissertation, University of Glasgow).

White, R. W., Jose, J. M., \& Ruthven, I. (2003). A task-oriented study on the influencing effects of query-biased summarisation in web searching. IPM '03, 39(5), 707-733.

Wilson, T. D. (1981). On user studies and information needs. Journal of documentation, 37(1), 3-15.

Zalesky, A., Fornito, A., \& Bullmore, E. (2012). On the use of correlation as a measure of network connectivity. NeuroImage, 60(4), 2096-2106. doi:http://dx.doi.org/10.1016/ j.neuroimage.2012.02.001 